\title{
Identification of epitaxial graphene domains and adsorbed species in ambient conditions using quantified topography measurements
}

Tim L Burnett, Rositsa Yakimova and Olga Kazakova

\section{Linköping University Post Print}

N.B.: When citing this work, cite the original article.

Original Publication:

Tim L Burnett, Rositsa Yakimova and Olga Kazakova, Identification of epitaxial graphene domains and adsorbed species in ambient conditions using quantified topography measurements, 2012, Journal of Applied Physics, (112), 5, 054308.

http://dx.doi.org/10.1063/1.4748957

Copyright: American Institute of Physics (AIP) http://www.aip.org/

Postprint available at: Linköping University Electronic Press

http://urn.kb.se/resolve?urn=urn:nbn:se:liu:diva-85205 


\title{
Identification of epitaxial graphene domains and adsorbed species in ambient conditions using quantified topography measurements
}

\author{
Tim L. Burnett, ${ }^{1, a)}$ Rositza Yakimova ${ }^{2}$ and Olga Kazakova ${ }^{1, b)}$ \\ ${ }^{1}$ National Physical Laboratory, Teddington TW11 0LW, United Kingdom \\ ${ }^{2}$ Linköping University, Linköping SE-581 83, Sweden
}

(Received 26 June 2012; accepted 27 July 2012; published online 6 September 2012)

\begin{abstract}
We discuss general limitations of topographical studies of epitaxial graphene in ambient conditions, in particular, when an accurate determination of the layers thickness is required. We demonstrate that the histogram method is the most accurate for measurements of small vertical distances $(<0.5 \mathrm{~nm})$ and generally should be applied to epitaxial graphene and similar types of samples in order to get the correct and reproducible values. Experimental determination of the step height between different domains of epitaxial graphene shows excellent agreement with the predicted values once the adsorption of a 2D monolayer is taken into account on top of the one layer graphene. In contrast to general limitations of AFM topography, electrostatic force microscopy imaging allows a straightforward identification of domains of epitaxial graphene of different thickness. [http://dx.doi.org/10.1063/1.4748957]
\end{abstract}

\section{INTRODUCTION}

Graphene, a single monolayer of graphite, is currently the subject of a massive research interest and an equally enormous number of publications due to its novel physical properties and vast potential in technological applications: a likely successor of silicon in post-Moore's law devices, biochemical sensors, $\mathrm{THz}$ applications, etc. ${ }^{1}$ Graphene has also been found to be extremely valuable for metrological applications, for example, exceptionally accurate measurements of the quantum Hall resistance quantization were demonstrated recently. ${ }^{2}$ In order to be economically viable and truly attractive for applications, large scale wafers of high quality graphene grown on insulating substrates are required. One of the most attractive routes is to grow graphene epitaxially from insulating $\mathrm{SiC}(0001)$ single crystals by solid-state graphitization of the substrate. ${ }^{3}$ Besides wafer-scale graphene production (up to 5 in. wafer), the method provides a possibility to better control the electronic properties of graphene via charge transfer through interaction with the substrate.

However, during the high-temperature annealing process the $\mathrm{SiC}$ substrate forms terraces with a typical height of 5-30 nm and eventually develops a complex surface morphology, which strongly depends on the growth conditions (temperature, gas atmosphere, pressure) as well as the initial mis-cut angle of the substrate. Most importantly, thermal decomposition of $\mathrm{SiC}$ is not a self-saturated process, which may result in the coexistence of graphene layers of various thicknesses. Thus, the $\mathrm{SiC}$ substrate significantly hinders straightforward identification and determination of the graphene layer thickness. For electronic applications, in particular, it is crucial to define the number of graphene layers

\footnotetext{
a) Present address: Material Science Centre at University of Manchester, Manchester M1 7HS, United Kingdom.

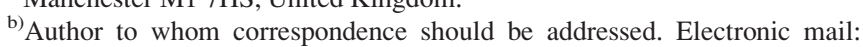
olga.kazakova@npl.co.uk.
}

precisely as, for example, one and two layers of graphene (1LG and 2LG) are characterised by a completely different band structure, represented by the absence/presence of the energy gap, respectively, which in turn defines the properties of devices. When morphology studies are performed in ambient conditions, the presence of water and various adsorbed species on the surface of graphene may further complicate the layer identification. ${ }^{4-6}$

Topography measurement using scanning probe microscopy (SPM) is a widely available and very versatile technique which has been extensively and successfully used for studies of the initial stages of graphitization on $\mathrm{SiC}^{7-9}$ as well as for investigation of linear defects in epitaxial graphene, i.e., ridges, wrinkles/puckers, pleats, etc., which are generally the result of the compressive strain between graphene and $\mathrm{SiC}$ during cooling from the annealing process. ${ }^{10}$ However, due to the complex morphology of the SiC substrate and inhomogeneity of the graphene growth, it is often very difficult, if not impossible, to define precisely the local thickness of the graphene studying the morphology alone. For example, a large variety of possible step configurations for $1 \mathrm{LG}$ and $2 \mathrm{LG}$ on SiC defined in Ref. 11 demonstrated that the height of a single layer can vary between 85 and $415 \mathrm{pm}$ depending on the stacking configuration and the conventional assumption of a $1 \mathrm{LG}$ height being $335 \mathrm{pm}$ is not appropriate for epitaxial graphene grown on SiC. Confidence can be obtained by combination of topography height and tapping phase images, ${ }^{9,11}$ which in many cases can distinguish between different graphene domains. Nevertheless, many experimental studies still rely on the commonly available and simple height measurements as the main source of identification of the graphene thickness, which often leads to ambiguous and irreproducible results, especially in ambient conditions. However, electrical modes of scanning probe microscopy (i.e., SKPM ${ }^{12,13}$ and electrostatic force microscopy $(\mathrm{EFM})^{14}$ ) have been recently successfully used to identify the number of layers in epitaxial graphene. 
The aim of this paper is to demonstrate some general limitations of topographical studies of epitaxial graphene in ambient conditions, in particular, the determination of the graphene layer thickness. We demonstrate that the histogram method is the most accurate for measurements of small $(<0.5 \mathrm{~nm})$ vertical distances. We also report that, if a correction is made to the measurements which assume a $2 \mathrm{D}$ film on top of the single layer graphene, the height measurements show excellent agreement with the theoretical values. We suggest that this film is likely to be a monolayer of adsorbed atmospheric water.

\section{METHOD}

The epitaxial graphene was prepared by sublimation of $\mathrm{SiC}$ and subsequent graphene formation on the Si-terminated face of a nominally on-axis $4 H-\mathrm{SiC}(0001)$ substrate at $2000^{\circ} \mathrm{C}$ and 1 bar argon gas pressure. These conditions allow a low rate of silicon losses from the surface (leaving carbon behind) while surface kinetics is favoured due to the high temperature. Since graphene coverage and thickness uniformity result from the progression of silicon sublimation and rearrangement of surface carbon atoms, it is crucial to have fast surface kinetics to avoid 2D island nucleation. Ultimately, the graphene quality can be controlled by several process variables. Details of the fabrication and structural characterization are reported elsewhere. ${ }^{15}$ The method has advantages over conventional UHV growth, potentially providing large areas of homogeneous graphene layers. ${ }^{3,7}$ Low magnetic field measurements established that the manufactured material was $n$-doped, owing to charge transfer from $\mathrm{SiC}$, with the measured electron concentration in the range $5-8 \times 10^{11} \mathrm{~cm}^{-2}$ and mobility of $\sim 2400-2800 \mathrm{~cm}^{2} \mathrm{~V}^{-1} \mathrm{~s}^{-1}$ at room temperature. ${ }^{2,16}$

All measurements were conducted on a Bruker Icon AFM in ambient conditions $\left(\mathrm{T}=20 \pm 0.5^{\circ} \mathrm{C}\right.$, relative humidity $\sim 43 \%$ ). Bruker SCM-PIT Pt-Ir coated probes with a force constant of $\sim 3 \mathrm{~N} / \mathrm{m}$ were used for all experiments. The typical radius of the tips was $\sim 10 \mathrm{~nm}$. Topography images of epitaxial graphene were recorded simultaneously with potential difference maps using amplitude and phase detection EFM. EFM is a two-pass method where the electrostatic force is measured between the charge on the sample and the tip (approximately a point dipole). This force is detected as a change in the resonant frequency, recorded as a phase shift, of the cantilever as it experiences either attractive or repulsive force. For these experiments, a lift height of $16 \mathrm{~nm}$ was used, using a range of DC biases, $-2 \mathrm{~V} \leq \mathrm{V} \leq+2 \mathrm{~V}$. Details of the method are described elsewhere. ${ }^{14}$ Standard precautions for sample handling and storage (i.e., in a polystyrene plastic storage box in a desiccator with humidity $\sim 25 \%$ ) were undertaken.

\section{EXPERIMENTAL RESULTS}

\section{A. Height measurements}

Throughout the paper, we use the following notation: IFL is the interface layer, which represents carbon-rich reconstructed $\mathrm{SiC}$ surface, $1 \mathrm{LG}$ is single and $2 \mathrm{LG}$ is double layer graphene and MLG refers to multilayer graphene. The sample is predominantly comprised of $1 \mathrm{LG}(\sim 60 \%$ of the total area) and $2 \mathrm{LG}$ is the balance of the area, whereas thicker MLG constitutes less than $2 \%$ of the total area. In this paper, we analyze a number of areas with a view to understanding the relative height of the graphene domains. $0 \mathrm{D}$ adsorbates are observed predominately on the $1 \mathrm{LG}$, leaving 2LG immaculately clean (Fig. 1). This decoration can often be used for easy distinction between the 1LG and 2LG domains. However, in some areas of the same sample such obvious decoration with $0 \mathrm{D}$ adsorbates does not occur as discussed below (Fig. 2).

The step heights between different domains of graphene have been measured using AFM via three different methods, namely: individual line profiles, averaged line profiles based on recording of 51 individual parallel lines, and histogram plots. Both image and step height analyses were undertaken in the SPIP ${ }^{\mathrm{TM}}$ image analysis software. ${ }^{17}$ In order to record appropriate step heights, it was crucial to adjust the tilt of the image to produce flattened steps with constant values across the graphene domains. However, the critical point of analysis is that, with an exception of tilting adjustment, no flattening procedures were applied to the images.

Figure 1 (top row from left to right) shows the topography, deflection error, and EFM phase images of the area where the $1 \mathrm{LG}$ domain is covered with $\mathrm{OD}$ adsorbates. Region A highlights an area including $1 \mathrm{LG}$ and $2 \mathrm{LG}$ domains, whereas region B shows a $1 \mathrm{LG}$ domain covering a $\mathrm{SiC}$ terrace. The step height measurements were performed in the highlighted regions. A comparison of experimental results performed by the three techniques for both areas is shown in Table I. For simplicity, Fig. 1 presents only the histogram and individual line profiles for region A and only the histogram and the average line profile for region $\mathrm{B}$.

As expected, the individual line method offers the least consistent result, e.g., the height of the step between 1LG and 2LG measured as 200-400 pm (Table I and Fig. 1, middle row). It should be noted that the minus sign in Table I corresponds to the situation when $1 \mathrm{LG}$ domain is below 2LG. The poor accuracy of height measurements is related to several factors which must be understood and controlled. First, height measurements of such small size are very difficult to perform accurately with a single line trace due to the mechanical noise floor imparting a large degree of noise to these line traces. Some statistics is required to help reduce this effect and averaged line profiles do offer an advantage, i.e., the same step as above was measured as $\sim 300 \mathrm{pm}$ (Table I). However, when there are features such as holes or in this case 0D adsorbates on top of the 1LG (Fig. 1), the value of the averaged line profile is compromised and may return an artificially low or high result. For these very small height steps close to the noise floor and due to the unavoidable and random nature (height, size, and position) of the adsorbates, each slightly different location of the line trace (single and averaged) produces different results. So, neither of these two techniques, i.e., single and averaged line trace methods, provides a very reliable measurement of the height between layers of graphene, first, due to the noise level and, second, due to the inhomogeneity of the surface. For the 

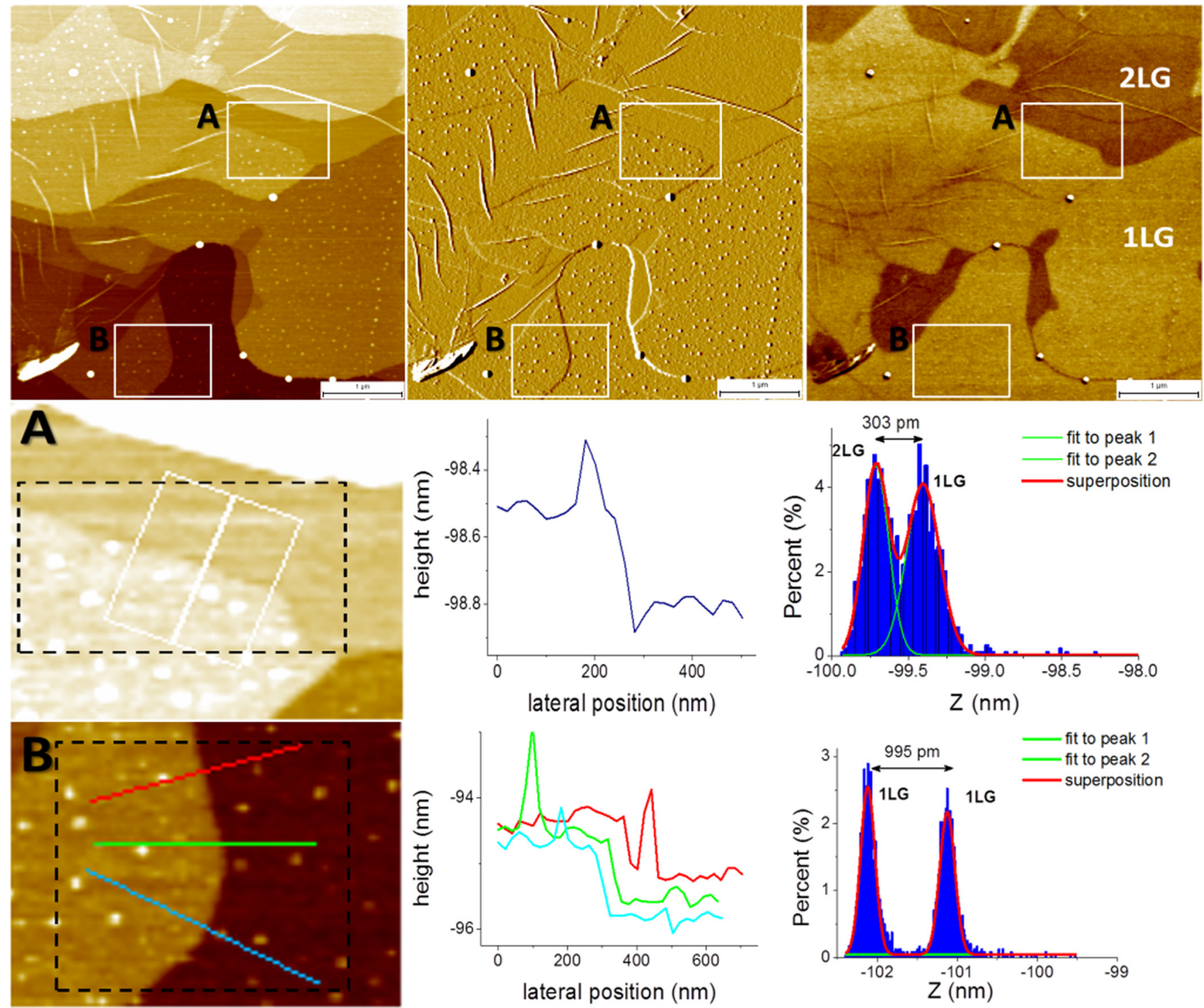

FIG. 1. Area of $1 \mathrm{LG}$ and $2 \mathrm{LG}$ domains, where the $1 \mathrm{LG}$ is decorated with $0 \mathrm{D}$ adsorbates. Top row left to right: topography, deflection error, and EFM phase images. The scale bar is $1 \mu \mathrm{m}$. Middle row: region A showing $1 \mathrm{LG}$ and $2 \mathrm{LG}$ domains. Bottom row: region B showing a $1 \mathrm{LG}$ domain only. For middle and bottom rows left to right: topography, step height profile, and histogram obtained from the dashed box in the topography image. The colored/white lines and frame highlight the location of corresponding profiles and the dotted black lines show the histogram areas. On both histograms: the green lines show the Gaussian fits to individual peaks, whereas the red line shows a multiple peak fit.

single line traces in Fig. 1, it can be seen that there is a large height variation related to surface defects, hence it is somewhat arbitrary to apply a best fit line to the step. As such these values become quite unreliable, the values quoted in Table I describe a maximum and minimum height that can reasonably be measured. This is deemed most appropriate for this method given the lack of repeatability in measurements made this way. The average line profiles combine multiple parallel single line profiles and with this method it is possible to extract repeatable measurements but a certain degree of uncertainty should be added as the technique includes all surface inhomogeneities in the averaged result. This is not a problem for surface that does not have such features and generally in our results this method shows good agreement with the histogram results. The error for measurements made this way is estimated to be $\pm 150 \mathrm{pm}$.

In this respect, the histogram method allows us to circumvent the influence of the adsorbates, i.e., the part of the histogram peak associated with the $0 \mathrm{D}$ adsorbates can be easily filtered and ignored. The method also provides the statistics required to minimise the noise effects. For example, the height of the step in region A (Fig. 1) was measured as $-303 \pm 50 \mathrm{pm}$ as the distance between two appropriately fitted histogram peaks (see also Table I). Whilst it is straightforward to appraise the appropriate degree of tilting using the line profile methods, it is not so simple for the histogram measurements, as the image needs to be adjusted for the whole XY plane for the region under analysis. We found that this can be achieved by ensuring that the histogram peaks associated with the different graphene domains fit closely to a symmetric Gaussian distribution. Fortunately, small inaccuracies in tilt correction in either $\mathrm{X}$ or $\mathrm{Y}$ direction do not result in large deviations from the properly tilted result.

From comparision of experimental results derived from the different methods in Table I, it is obvious that each 


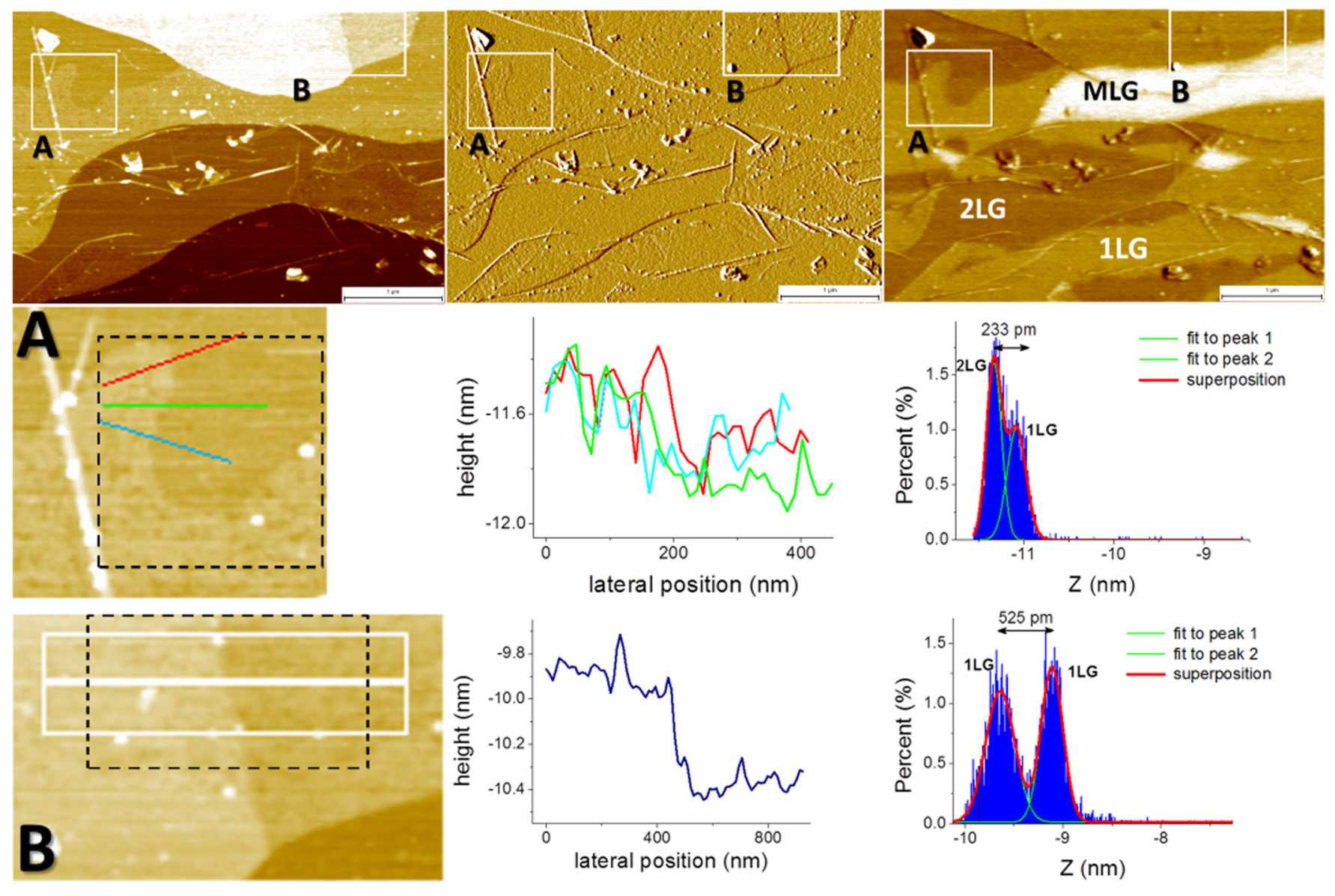

FIG. 2. Area of $1 \mathrm{LG}$ and $2 \mathrm{LG}$ domains without significant $0 \mathrm{D}$ adsorbate decoration. Top row left to right: topography, deflection error, and EFM phase images. The scale bar is $1 \mu \mathrm{m}$. Middle row: region A showing $1 \mathrm{LG}$ and 2LG domains. Bottom row: region B showing a 1LG domain only. For middle and bottom rows left to right: topography, step height profile, and histogram obtained from the dashed box in the topography image. The colored/white lines and frame highlight the location of corresponding profiles and the dotted black line shows the histogram area. On both histograms: the green lines show the Gaussian fits to individual peaks, whereas the red line shows a multiple peak fit.

method offers quite different results. The risk is that with each of the different methods presented it is possible to "generate" a spurious height value, especially working with extremely small heights typically well below $0.5 \mathrm{~nm}$. Of all of these measurement techniques, the histogram is the most reliable with repeated measurements giving a good accuracy. This is provided by the statistics inherent to the technique and also because inhomogeneities in the samples surface, i.e., surface debris or holes, can be effectively circumvented as they are separated out in the creation of the histogram. The main difficulty here is to assure that the appropriate level of tilting has been reached. We show that the fitting of Gaussian curves to the histogram is one way of effectively checking this. The uncertainty in the histogram measurements is most compromised by systematic errors which are estimated at $\pm 50 \mathrm{pm}$.

TABLE I. Comparison of experimental results and model predictions for the step height for Figs. 1 and 2 . All height values are in picometers and the minus sign signifies that the 1LG domain is below the $2 \mathrm{LG}$ domain. The model results show a range of possible options for the height between the graphene domains as theorised by the referenced works. The corrected values simply add $400 \mathrm{pm}$ to the $1 \mathrm{LG}$ to give recalculated step heights.

\begin{tabular}{|c|c|c|c|c|}
\hline & \multicolumn{2}{|l|}{ Fig. 1 (with 0D adsorbates) } & \multicolumn{2}{|l|}{ Fig. 2 (no 0D adsorbates) } \\
\hline & Region A $1 \mathrm{LG}$ to $2 \mathrm{LG}$ & Region B $1 \mathrm{LG}$ to $1 \mathrm{LG}$ & Region A $1 \mathrm{LG}$ to $2 \mathrm{LG}$ & Region B $1 \mathrm{LG}$ to $1 \mathrm{LG}$ \\
\hline Single Line & -200 to -400 & 800 to 1200 & +200 to +500 & 400 to 700 \\
\hline Averaged Line & -300 & 1050 & +200 & 500 \\
\hline Histogram & -303 & 995 & +233 & 525 \\
\hline $\begin{array}{l}\text { Model } 1 \\
\left(\text { Filleter } \text { et } a l .{ }^{11}\right)\end{array}$ & $-915,-665,-415,-165,+85,+335$ & 1000 & $-915,-665,-415,-165,+85,+335$ & 500 \\
\hline $\begin{array}{l}\text { Model 1-corr } \\
\text { Model } 2\end{array}$ & $-515,-265,-15,+235,+485,+735$ & 1000 & $-515,-265,-15,+235,+485,+735$ & 500 \\
\hline$\left(\right.$ Hass et al. $\left.{ }^{19}\right)$ & $-925,-673,-421,-169,+83,+335,+587$ & 1008 & $-925,-673,-421,-169,+83,+335,+587$ & 504 \\
\hline Model 2-corr & $-525,-273,-21,+231,+483,+735$ & 1008 & $-525,-273,-21,+231,+483,+735$ & 504 \\
\hline
\end{tabular}




\section{B. Height steps measured between 1LG and 2LG domains}

The sample under study predominantly consists of $1 \mathrm{LG}$ and $2 \mathrm{LG}$ as shown by Raman and EFM measurements. ${ }^{6}$ When the solid-state graphitization method is used, the growth of graphene occurs by thermal decomposition of $\mathrm{SiC}$ "downwards" into the substrate by transforming the $\mathrm{SiC}$ layers into graphene. It is generally accepted that 3 layers of $\mathrm{SiC}$ are required to create a single layer of graphene. ${ }^{18}$ Thus, it is very common to have the $1 \mathrm{LG}$ depressed with respect to the substrate or thicker layers of graphene which require more layers of $\mathrm{SiC}$ to be created (Figs. 1 and 2).

Despite the intensive studies of epitaxial graphene on $\mathrm{SiC}(0001)$, the nature and properties of the IFL are not very well understood. However, it is believed that this layer plays a defining role in the electronic properties of epitaxial graphene. At present, the consensus is that IFL is responsible for the $(3 \sqrt{ } 6 \times 3 \sqrt{ } 6) \mathrm{R} 30^{\circ}$ reconstruction of $\mathrm{SiC}(0001)$ and essentially it is a carbon-rich layer covalently bonded to the substrate. ${ }^{19}$ This implies that IFL is not a simple relaxed bulk termination of the SiC surface and the interface reconstruction is complex and may extend relatively deep into the bulk. The incomplete understanding of the IFL inevitably causes different interpretation of its thickness. For example, the value $\mathrm{t}_{\mathrm{IFL}}=232 \mathrm{pm}$ was obtained by Hass et al. ${ }^{19}$ using the surface $\mathrm{x}$-ray reflectivity method. On the other hand, values of $\mathrm{t}_{\mathrm{IFL}}=250 \mathrm{pm}$ and $\mathrm{t}_{\mathrm{IFL}}=240 \pm 30 \mathrm{pm}$ were predicted by Filleter et al. ${ }^{12}$ and Emtsev et al., ${ }^{18}$ respectively, using a conventional layer attenuation model. Recently, it was argued that the thickness of the IFL is not constant but dependent on the thickness of the graphene layer on top varying in a very broad range of $\mathrm{t}_{\mathrm{IFL}}=150-900 \mathrm{pm}^{20}$

Initially, we consider the height of $\mathrm{SiC}$ terraces, $\mathrm{t}_{\mathrm{SiC}}$, under $1 \mathrm{LG}$ (region B, Figs. 1 and 2). The step height on the terraces from $1 \mathrm{LG}-1 \mathrm{LG}$ across a step always appears as a multiple of $250 \pm 50 \mathrm{pm}$, which is in very good agreement to the expected height of one layer of $\mathrm{SiC}$ as predicted both by Filleter et al. $\left(\mathrm{t}_{\mathrm{SiC}}=250 \mathrm{pm}\right)$ and Hass et al. $\left(\mathrm{t}_{\mathrm{SiC}}=252 \mathrm{pm}\right)$, see Table I. This was tested over a large number of images taken from different locations of the same sample and remained extremely consistent and reliable when using the histogram method with proper tilting.

We also investigated the height between 1LG and 2LG (region A) and compared these measurements with existing models. Below we contemplate two possible scenarios taking into account thickness values after Refs. 12 and 19:

(1) With a SiC and IFL layer height of $t_{\mathrm{SiC}}=\mathrm{t}_{\mathrm{IFL}}=250 \mathrm{pm}$ and nominal height of $1 \mathrm{LG}$ being $335 \mathrm{pm}$, it is clear that the real step height as measured by AFM will strongly depend on the exact stack configuration. Filleter et al. ${ }^{12}$ demonstrated that for combinations of IFL, 1LG, and 2LG the step height values could be $-415,-165,+85$, and $+335 \mathrm{pm}$ giving several possible combinations where the total height is $<0.5 \mathrm{~nm}$. Further developing the analysis by Filleter et al. ${ }^{12}$ and considering only the case when 1LG is higher than 2LG (as is the case in
Figs. 1 and 2), the possible step heights between IFL and 1LG are: $-915,-665,-415$, and $-165 \mathrm{pm}$.

(2) Hass et al. ${ }^{19}$ considered the thicknesses of $\mathrm{SiC}$ layer being of $t_{\mathrm{SiC}}=252 \mathrm{pm}$, IFL of $\mathrm{t}_{\mathrm{IFL}}=232 \mathrm{pm}$, the first layer of graphene of $350 \mathrm{pm}$ and subsequent graphene layers as $335 \mathrm{pm}$ thick. Thus, the possible heights between $1 \mathrm{LG}$ and $2 \mathrm{LG}$, with $1 \mathrm{LG}$ being higher, work out as $-925,-673,-421$, and $-169 \mathrm{pm}$.

All values between $1 \mathrm{LG}$ and $2 \mathrm{LG}$ as well as across SiC terraces expected from these models are presented in Table I. As it can be seen from the comparison, experimental measurements between different domains, i.e., $1 \mathrm{LG}$ and $2 \mathrm{LG}$, the height steps do not match the expected values, despite careful tilting and the use of the histogram method to measure the height. For example, the experimentally obtained height of -303 pm (Fig. 1, Table I) shows no direct match to expected values predicted by either Filleter or Hass models with the nearest result over 100 pm away.

We can very effectively account for this discrepancy by assuming that the exposed 1LG across the whole sample is covered by a thin continuous "adsorbate" layer. The thickness of this 2D adsorbate layer is consistent over the sample. This is based on the fact that the height of the $\mathrm{SiC}$ terraces always appears as multiples of $250 \mathrm{pm}$ regardless of the graphene layer thickness that covers the terrace. This 2D adsorbate film should not be mixed up with OD adsorbates which can be clearly seen on topography images (Fig. 1). When a value of $400 \mathrm{pm}$ was added to the height of the $1 \mathrm{LG}$ only, for the numerous locations studied in this work, we obtained a result that agreed closely with the values expected from models. The corrected model values in Table I take into account this "adsorbed" layer which clearly matches the experimental results within error. We suggest that this adsorbed layer is likely to be a monolayer of water based on the ambient environment of the measurements and the thickness of a monolayer of water on graphene should be approximately $400 \mathrm{pm}$ according to previous studies. ${ }^{21,22}$ Fig. 3(a) shows a schematic representation of the experimental case depicted in Fig. 1. There is also some evidence in the literature as to why such a layer would form only on the 1LG as this should be significantly more hydrophilic than thicker
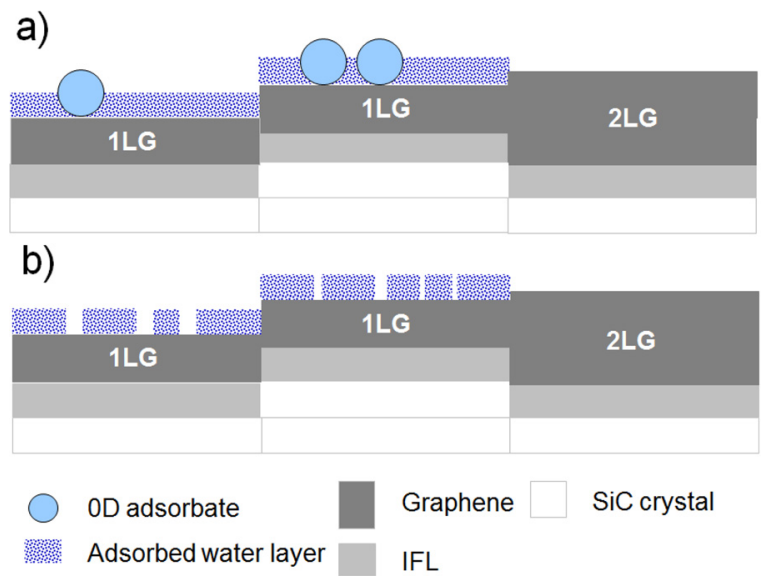

FIG. 3. Schematic diagram showing the arrangement of graphene, IFL, 2D, and $0 \mathrm{D}$ adsorbates corresponding to (a) Fig. 1 and (b) Fig. 2. 
graphene domains as the surface properties are strongly influenced by the hydrophilic SiC substrate. ${ }^{23}$

The use of histograms for height measurements accompanied by the consideration of an adsorbed layer has allowed for accurate measurements of the height between epitaxial graphene domains to be made. This is usually precluded by the complex surface morphology. The corrected heights now agree very well with those described by Hass et al. ${ }^{19}$ and Filleter et al. ${ }^{12}$ Our measurements show that the adsorbed layer is very stable as it exists almost uniformly across the sample and a simple subtraction of this additive layer has in this case allowed for identification of graphene layers based on height measurements in epitaxial graphene. This has been tested across multiple locations on the sample with excellent agreement. It should be noted that the nature and thickness of the adsorbed layers will be strictly related to the sample history and, therefore will vary for different samples. However, the uniformity and stability of the adsorbed layer, which we think could be water, suggest that a similar approach may be applicable to many other epitaxially grown graphene samples where individual layers are otherwise difficult to identify.

\section{Height steps measured in regions of discontinuous adsorbed layer}

For the same sample, there were a few regions $(<25 \%$ of surface) which were more difficult to interpret. A typical example is shown in Fig. 2. These are relatively rare regions across the sample and are visibly different from the general situation described with respect to Fig. 1. First, the lack of OD adsorbates is clear and, furthermore, holes can be observed in this discontinuous adsorbed layer. Such regions are often associated with surface debris typically occurring during the sample growth (Fig. 2). Comparing the magnified images of regions $\mathrm{A}$ and $\mathrm{B}$ in Figs. 1 and 2, the discontinuous nature of the adsorbed layers in Fig. 2 can be clearly seen. In this case, the adsorbed layer on top of 1LG is full of holes (Fig. 2, middle and bottom rows), whereas the graphene surface appears to be homogeneous in Fig. 1 and for 2LG in Fig. 2. Regions such as those shown in Fig. 2 still fit the model which assumes a continuous water layer of $400 \mathrm{pm}$ thickness to a good degree of accuracy (see Table I). The schematic diagram of this case is shown in Fig. 3(b). There is some spread in the histogram data in Fig. 2 but the presence of a peak related to the "height" of the holes is not obvious. It is suggested that the small total area of the holes has precluded this analysis. Additionally, the small lateral dimensions, $<50 \mathrm{~nm}$, of the holes, as evidenced by the line trace data, show that reliable height measurements cannot be reliably made on these features due to a combination of a convolution of the tip shape and the image resolution.

The SiC terrace height measured in the location of $1 \mathrm{LG}$ for the region in Fig. 2 also agrees very closely to the predicted value and adds confirmation to the assertion that the adsorbate layer has a very consistent thickness across the entire sample, despite its different appearance, allowing for direct measurement of the SiC terraces whose height is always equal to multiples of the layer thickness $\left(\mathrm{t}_{\mathrm{SiC}}\right)$.

Overall, a large number of AFM images were taken across the sample with the total area of $7 \times 7 \mathrm{~mm}^{2}$. Within each image, recorded height measurements consistently fitted the model when the adsorbed layer on top of the $1 \mathrm{LG}$ was taken into account.

\section{Nature of the adsorbed layer}

Preliminary studies using secondary ions mass spectroscopy (SIMS) show the presence of polydimethylsiloxane (PDMS) as the major contaminant with traces of erucamide and dimyristoylphophatidylcholine. Based on previous studies showing the formation of siloxane films on carbon surfaces, it is thought that these species are responsible for the $0 \mathrm{D}$ adsorbates on the sample surface. This is due to the fact that the process of siloxane adsorption starts from formation of individual islands, and with increased surface coverage a densely packed monolayer of islands forms. ${ }^{24,25}$ These compounds most likely originate from the environment of plastic storage containers, gloves, and numerous other applications where PDMS is used as a mould release agent. No water was observed or expected due to the fact that the SIMS measurements were carried out in very high vacuum $\left(10^{-8}\right.$ mbar).

These results signify the importance of ensuring true homogeneity of the sample, as any species adsorbed on to the graphene surface will potentially affect the fabrication and performance of devices. It should be emphasised that all reasonable care was taken in the handling and storage of the samples through the use of desiccators and manipulation with tweezers.

As described above, identification of graphene domains on topography images is a significant challenge, although we show that it is generally appropriate to use a histogram method and taking into account an adsorbed layer on top of the $1 \mathrm{LG}$ can reconcile this. In the present study, the thickness and overall appearance of the 2D adsorbate layer is generally consistent with a monolayer of water, although other possibilities cannot be completely excluded at the moment. The relevant chemical studies should resolve the question and are being performed. Yet, consideration of the additional 2D adsorbate layer provides excellent agreement between our direct AFM measurements and several of the current models of height expected between graphene domains. We also demonstrate that difficulties in identification of the number of layers in epitaxial graphene in ambient conditions from topography measurements can to a large extent be successfully overcome using EFM technique and the adsorbed water layer does not preclude the identification of graphene domains from EFM phase contrast (see Figs. 1 and 2, top row, right images).

\section{SUMMARY}

In this paper, we showed that the histogram method is the most reliable for measurements of small vertical distances $(<0.5 \mathrm{~nm})$ and should be applied to epitaxial graphene and similar types of samples in order to get correct and reproducible values. Fitting of the histogram to a Gaussian curve is a way of checking appropriate flattening of the histogram data. We show that height measurements are in excellent agreement with current models, once we take into account an adsorbed layer that forms on top of the $1 \mathrm{LG}$. The adsorbed layer is very stable in ambient conditions and characterised by a uniform thickness of $400 \mathrm{pm}$ across all areas 
investigated on the sample. The thickness of the additional adsorbate layer is consistent with the thickness of a monolayer of water on graphene. The nature of the $0 \mathrm{D}$ adsorbates is generally ascribed to polydimethylsiloxane and other organic compounds originating from standard plastic packaging and handling. We also demonstrate that EFM imaging allows a straightforward identification of epitaxial graphene domains of different thickness. Our studies highlight the degree to which the graphene surface is susceptible to adsorption of species in ambient conditions. It is imperative to understand this for processing and applications of epitaxial graphene, as the adsorbed species will modify the graphene properties.

\section{ACKNOWLEDGMENTS}

This work has been funded by the Strategic Research (NPL) under Project 114753, EMRP under Project 115367 (MetMags) and EU FP7 Project "ConceptGraphene." We are very grateful to Alexander Tzalenchuk and Ian Gilmore for useful discussions and Rasmus Havelund for SIMS measurements.

${ }^{1}$ K. Novoselov, Rev. Mod. Phys. 83, 837-849 (2011).

${ }^{2}$ A. Tzalenchuk, S. Lara-Avila, A. Kalaboukhov, S. Paolillo, M. Syväjärvi, R. Yakimova, O. Kazakova, T. J. B. M. Janssen, V. Fal'ko, and S. Kubatkin, Nat. Nanotechnol. 5, 186-189 (2010).

${ }^{3}$ C. Virojanadara, M. Syväjarvi, R. Yakimova, L. Johansson, A. Zakharov, and T. Balasubramanian, Phys. Rev. B 78, 1-6 (2008).

${ }^{4}$ J. Moser, A. Verdaguer, D. Jiménez, A. Barreiro, and A. Bachtold, Appl. Phys. Lett. 92, 123507 (2008).

${ }^{5}$ A. Verdaguer, M. Cardellach, J. J. Segura, G. M. Sacha, J. Moser, M. Zdrojek, A. Bachtold, and J. Fraxedas, Appl. Phys. Lett. 94, 233105 (2009).
${ }^{6}$ T. L. Burnett, J. Patten, and O. Kazakova, e-print arXiv:1204.3323v1.

${ }^{7}$ K. V. Emtsev, A. Bostwick, K. Horn, J. Jobst, G. L. Kellogg, L. Ley, J. L. McChesney, T. Ohta, S. Reshanov, J. Röhrl, E. Rotenberg, A. K. Schmid, D. Waldmann, H. B. Weber, and T. Seyller, Nat. Mater. 8, 203-207 (2009).

${ }^{8}$ M. Bolen, S. Harrison, L. Biedermann, and M. Capano, Phys. Rev. B 80, $1-9$ (2009).

${ }^{9}$ F. J. Ferrer, E. Moreau, D. Vignaud, D. Deresmes, S. Godey, and X. Wallart, J. Appl. Phys. 109, 054307 (2011).

${ }^{10}$ G. Prakash, M. L. Bolen, R. Colby, E. A. Stach, M. A. Capano, and R. Reifenberger, New J. Phys. 12, 125009 (2010).

${ }^{11}$ N. Camara, A. Tiberj, B. Jouault, A. Caboni, B. Jabakhanji, N. Mestres, P. Godignon, and J. Camassel, J. Phys. D: Appl. Phys. 43, 374011 (2010).

${ }^{12}$ T. Filleter, K. V. Emtsev, T. Seyller, and R. Bennewitz, Appl. Phys. Lett. 93, 133117 (2008).

${ }^{13}$ T. Filleter and R. Bennewitz, Phys. Rev. B 81, 1-7 (2010).

${ }^{14}$ T. Burnett, R. Yakimova, and O. Kazakova, Nano Lett. 11, 2324-2328 (2011).

${ }^{15}$ R. Yakimova, C. Virojanadara, D. Gogova, M. Syväjärvi, D. Siche, K. Larsson, and L. I. Johansson, Mater. Sci. Forum 645-648, 565-568 (2010).

${ }^{16}$ V. Panchal, K. Cedergren, R. Yakimova, A. Tzalenchuk, S. Kubatkin, and O. Kazakova, J. Appl. Phys. 111, $07 E 509$ (2012).

${ }^{17}$ See http://www.imagemet.com/ for image and height analyses.

${ }^{18}$ P. Lauffer, K. V. Emtsev, R. Graupner, T. Seyller, and L. Ley, Phys. Rev. B 77, 1-10 (2008)

${ }^{19}$ J. Hass, J. Millán-Otoya, P. N. First, and E. H. Conrad, Phys. Rev. B 78, 205424 (2008).

${ }^{20}$ J. H. Park, W. C. Mitchel, H. E. Smith, L. Grazulis, and K. G. Eyink, Carbon 48, 1670-1673 (2010).

${ }^{21}$ K. Xu, P. Cao, and J. R. Heath, Science (N.Y.) 329, 1188-1191 (2010).

${ }^{22}$ J. Shim, C. H. Lui, T. Y. Ko, Y.-J. Yu, P. Kim, T. Heinz, and S. Ryu, Nano Lett. 12, 648 (2012).

${ }^{23}$ J. Rafiee, X. Mi, H. Gullapalli, A. V. Thomas, F. Yavari, Y. Shi, P. M. Ajayan, and N. A. Koratkar, Nat. Mater. 11, 217 (2012).

${ }^{24}$ V. Koutsos, H. Haschke, M. J. Miles, and F. Madani, MRS Proc. 734, B1.6 (2002).

${ }^{25}$ F. Madani-Grasset, N. T. Pham, E. Glynos, and V. Koutsos, Mater. Sci. Eng. B 152, 125-131 (2008). 